OPEN ACCESS

Edited by:

Ghaleb Hamad Alnahdi, Prince Sattam Bin Abdulaziz

University, Saudi Arabia

Reviewed by:

W. L. Quint Oga-Baldwin,

Waseda University, Japan

Donald Sharpe,

University of Regina, Canada

Honglei Gu,

Hunan Normal University, China

*Correspondence:

Abdullah Alamer

aa.alamer@kfu.edu.sa

orcid.org/0000-0003-4450-0931

Specialty section:

This article was submitted to

Quantitative Psychology

and Measurement,

a section of the journal

Frontiers in Psychology

Received: 28 June 2021 Accepted: 02 September 2021 Published: 30 September 2021

Citation:

Alamer A (2021) Construct Validation of Self-Determination Theory in Second Language Scale: The Bifactor Exploratory Structural

Equation Modeling Approach.

Front. Psychol. 12:732016. doi: 10.3389/fpsyg.2021.732016

\section{Construct Validation of Self-Determination Theory in Second Language Scale: The Bifactor Exploratory Structural Equation Modeling Approach}

\author{
Abdullah Alamer ${ }^{1,2 *}$ \\ ${ }^{1}$ Department of English, Imam Muhammad Ibn Saud Islamic University, Al-Ahsa, Saudi Arabia, ${ }^{2}$ Department of English, King \\ Faisal University, Al-Ahsa, Saudi Arabia
}

The present study aimed to assess the construct validity of the Self-Determination Theory in Second Language Scale (SDT-L2; Alamer, 2021). The study involved 266 undergraduate students learning English as a second language (L2) in Saudi Arabia. The factorial structure of the SDT-L2 was examined using the advanced bifactor-Exploratory Structural Equation Modeling (ESEM) method. The scale provided adequate composite reliability and the bifactor ESEM provided unique details about the multidimensionality of the scale which accounted for the specific constructs (i.e., intrinsic, identified, introjected, and external regulations) and the general constructs (i.e., autonomous motivation and controlled motivation), and allowed for assessment of convergent and discriminant validity. Predictive validity was established by showing that autonomous motivation significantly predicted L2 performance, while controlled motivation did not. Overall, the study demonstrated the usefulness of the bifactor ESEM for construct validation purposes and the results showed that SDT-L2 is a valid scale to assess students' L2 motivation based on SDT perspective.

Keywords: construct validation, bifactor exploratory structural equation modeling, convergent validity, discriminant validity, langauge learning, exploratory structural equation modeling (ESEM), confirmatory factor analyses (CFA), self-determination theory (SDT)

\section{INTRODUCTION}

Self-determination theory (SDT) is a macro theory of motivation that has been applied to several life domains to understand what drives individuals to pursue their goals (Ryan and Deci, 2000, 2020). SDT has demonstrated valuable and valid insights about the motivation of learners who learn a second language (L2) in different language contexts (Noels et al., 1999; Alamer, 2021; Alamer and Almulhim, 2021). Essentially, SDT posits that motivation is multidimensional and depends on the extent to which individuals originate their behavior from within (Ryan and Deci, 2000). The theory maintains the existence of two general types of motivation, autonomous motivation, and controlled motivation with each having two sub-types of regulations. Autonomous motivation refers to the quality of individuals' motivation being volitional. At the extreme of autonomous motivation is intrinsic regulation which reflects language learners' inherent inclination toward carrying out the language tasks. Next comes identified regulation which is seen as an extrinsic type 
of autonomous motivation, and reflects the value and importance language learners attach when carrying out the language tasks. In contrast, controlled motivation refers to the type of motivation that is controlled by external circumstances and has two subtypes of regulations. At the extreme of controlled motivation is external regulation which reflects language learners' desire to get rewards or avoid punishments in carrying out language tasks. Next comes introjected regulation which is seen and partially internalized into the self (i.e., less controlled) and reflects the internal pressure language learners have in carrying out language tasks such as avoiding shame, guilt for failing, or anxiety. In the L2 domain, researchers assessed the relation between these regulations and different language outcomes including willingness to communicate (Watanabe, 2011), engagement (Oga-Baldwin and Nakata, 2017; Dincer et al., 2019; Noels et al., 2019), positive affect (Alamer and Lee, 2019), students' GPA (Noels et al., 1999), and attainment of the vocabulary (Alamer, 2021). These studies confirmed the positive relationships between the more autonomous types of motivation and the outcomes while showing that controlled types of motivation are either unrelated or negatively related to the outcomes.

One of the earliest scales presented to the field that assesses SDT regulations is perhaps Noels et al. (1999) scale. Based on the SDT literature, the researchers used correlation analysis to provide preliminary evidence of scale reliability and validity. The field has benefited from using this scale for different research contexts, but recent research showed that there is room for improvement. For example, Alamer and Lee (2019) pointed out that introjected regulation consistently results in weak reliability in the literature (Cronbach's alpha $(\alpha)$ as low as 0.59 ) because the construct is measured by only two items. Collier (2020) indicated that factors in CFA that are assessed by only two items usually yield measurement issues in the solution including weak reliability estimates. Although Noels et al. (1999) scale has gone through standard CFA (Ardasheva et al., 2012), its reliability estimates were rather low (again, as low as 0.58 in three distinct samples) and the correlation between the constructs was inflated because of the constraints CFA imposes. Critically, the correlation between intrinsic regulation and external regulation was positive and large $(r=0.87)$ which has been increased substantially from $(r=0.55)$ in the EFA (i.e., $\Delta r=0.32)$, thus the CFA results contradict the SDT distinction between the two constructs. Further, the formulation of SDT postulated the co-existence of two overarching constructs (i.e., autonomous motivation vs. controlled motivation) with each having two sub-types of regulations. This formulation cannot be captured statistically by correlation or the typical Exploratory Factor Analysis (EFA) or standard CFA because they do not account for the assessment of global constructs. For these reasons, Alamer (2021) has developed a modified scale, named SDT-L2 scale, based on the work of Noels et al. (1999) and presented preliminary evidence of the construct validity. The SDT-L2 scale has four subscales representing the four regulations with each having 5 items equally. Alamer (2021) evaluated the construct validity and reliability of the scale by using higher-order CFA. The higher-order model was chosen because it permits the inclusion of second-order factors that affects first-order factors (e.g., autonomous motivation as a second-order factor affecting the first-order factors, intrinsic and identified regulations). The analysis provided satisfactory results and the study is perhaps the first to account for the global constructs of autonomous motivation and controlled motivation in a measurement model.

Nonetheless, recent advancement in construct assessment has brought a relatively new method called Exploratory Structural Equation Modeling (ESEM) that integrates the best of CFA and EFA in one analysis (Marsh et al., 2009; Morin et al., 2013; Alamer and Marsh, 2022). ESEM is similar to EFA as it allows items to cross-load on all factors involved in the analysis and differs from the EFA as it takes on the features of Structural Equation Modeling (SEM) such as evaluating model fit indices, assessing and allowing to correlate the measurement errors, and allowing for different model specifications to be included [see Alamer and Marsh (2022) who introduced the method to the L2 field]. In almost all multidimensional studies, ESEM presents a better fit to the data because of the flexibility and less restrictive system it has over the CFA (see Morin et al., 2016 for details). Within the CFA and ESEM frameworks, a bifactor model can be evaluated (Reise et al., 2010). Bifactor models are alternatives to the higherorder models which postulate the co-existence of the general factors along with specific factors (Morin et al., 2016). In bifactor CFA, the items are loaded on their specific factors and on the general factors they presume to correspond to, while in bifactor ESEM all items loaded on the specific factors while loading on the general factors they presume to represent. In both bifactor CFA and ESEM, the factors are set to be orthogonal (i.e., correlations are set to be zero). Figure 1 illustrates visually the difference between the two models. Howard et al. (2018) state that bifactor models should be selected over the higher-order models unless strong conceptual justifications are present. Thus, we take on this perspective in assessing the construct validity of SDT-L2 scale.

Multidimensional research often reported that bifactor models (whether in ESEM or CFA) provide a better model fit to the data and capture the co-existence of the global (general) factor meaningfully (Reise et al., 2010; Morin et al., 2016). This is true with studies that evaluate the SDT subscales, wherein researchers observe that bifactor models are superior to the standard solutions. For example, Howard et al. (2018) has investigated the multidimensionality of SDT scale for workplace among Canadian employees and tested its continuum structure. The researchers achieved this by contrasting the results of bifactor CFA with those of bifactor ESEM and the findings showed that the bifactor CFA solution did not fit the data appropriately whereas the bifactor ESEM did. The researchers then evaluated a structural bifactor ESEM to examine how the factors are related to outcomes such as employees' positive affect, and the results were in line with the SDT proposition. The authors concluded that bifactor ESEM "provides an alternative approach that allows for the simultaneous consideration of the global quantity of self-determined motivation together with all qualitative variations along the SDT continuum in a single model not tainted by multicollinearity" (Howard et al., 2018, p. 22).

The SDT-L2 scale (Alamer, 2021) has been successfully introduced to the language learning field to assess L2 learners' 

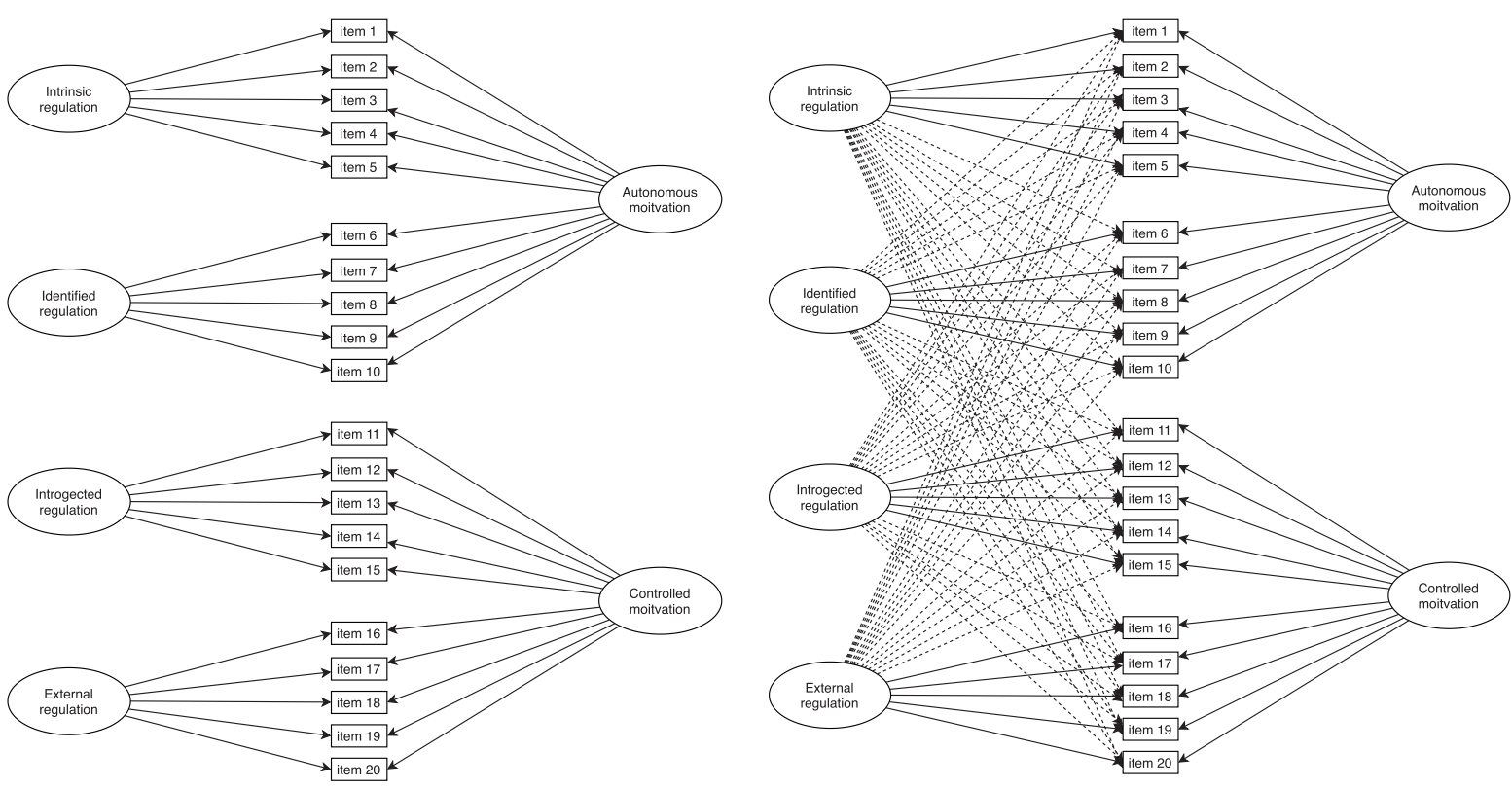

FIGURE 1 | Visual representation of the bifactor CFA (on the left) and bifactor ESEM (on the right).

motivation based on the SDT (Alamer and Lee, 2021), however, the internal structure of this scale has not been replicated using the more appropriate statistical tool such as the bifactor ESEM to capture the appearance of the global factors (autonomous motivation and controlled motivation). Thus, the present study aimed to replicate the construct validity and predictive validity of the SDT-L2 scale in a more appropriate manner. To do so, the author first compare the results of the bifactor CFA and bifactor ESEM in terms of goodness-of-fit. Second, the author proceed with the solution that shows a better fit and tests its predictive validity for language outcome (i.e., L2 performance). Based on previous studies (e.g., Howard et al., 2018), it is hypothesized that bifactor ESEM would fit the data better and would be able to capture the variance in both specific and global factors meaningfully. In addition, as it is theoretically and empirically supported (Alamer and Lee, 2019; Dincer et al., 2019; Noels et al., 2019), it is postulated that in the structural bifactor ESEM, autonomous motivation would positively predict scores in L2 performance, while controlled motivation would be negatively or unrelated to L2 performance.

\section{METHODS}

The study sample consisted of 266 Saudi undergraduate students who learn English as the L2 in Saudi universities. The participants were aged between 18 and 22 years, with a mean age of 19.3 years $\left(\mathrm{SD}_{\mathrm{age}}=0.46\right)$. The sample was $42 \%$ male and $58 \%$ female. A convenience sample strategy was used, and students were invited through email to participate in an online questionnaire made by Google Forms. Students who do not want to participate or want to withdraw while participating were asked to refrain from completing the questionnaire and simply close the webpage.

\section{Measures}

\section{The Self-Determination Theory in Second Language Scale}

The SDT-L2 scale comprises 20 items measuring 4 specific constructs of SDT, intrinsic regulation, identified regulation, introjected regulation, and external regulation (see the Supplementary Appendix for the full scale). The scale was designed in a 5-point Likert-type response format. Each subscale consists of five items [and the four subscales represent the Two overarching constructs (i.e., autonomous vs. controlled)]. Participants Were asked to ponder the question "Why Are you learning English?" and then indicate the extent to which they agreed With the statements that followed. Example items Are as follows: For intrinsic regulation, "because I enjoy learning English"; For identified regulation, "because learning English Is important for my personal growth"; For introjected regulation, "because I Would feel ashamed if I am Not successful in English learning like my friend (s)/family"; And for external regulation, "because I just want to pass the English exam."

\section{L2 Performance}

Students' language performance was assessed using two measures. The first is their students' grade point averages (GPA) in their English courses. Students' scores on this measure ranged from 1 to 5 , with 5 representing excellent progress in the language courses. The units of English lessons include subjects of Reading, Writing, Speaking, Listening, Vocabulary, and Phonetics. The second measure was students' effort in learning the L2. Five items taken from Gardner's (2010) measure of effort were used. An example item is "I really work hard to learn English.” 


\section{Statistical Analyses}

To assess the validity and reliability of the SDT-L2 scale, The author used two software tools: Jamovi (The Jamovi Project, 2019) and Mplus 8.1 (Muthén and Muthén, 1998). First, the author tested univariate normality by inspecting the skewness and kurtosis using the " $+2 /-2$ " and $+10 /-10$ guidelines, respectively (Collier, 2020). The author obtained the reliability of the constructs using composite reliability (CR). CR (also called coefficient omega) considers the factor loadings of the observed variables and their measurement errors in the calculation. Hence, this reliability measure is obtained from the measurement model (see Collier, 2020 for details). To test the goodness-of-fit, the $\chi^{2}$ and its $p$-value were evaluated. Alternative measures were also used such as the comparative fit index (CFI), the TuckerLewis index (TLI), the root mean square error of approximation (RMSEA), and the standardized root mean square residual (SRMR) were used. CFI and TLI values that are in the region of 0.95 are indicative of a good model fit. Values smaller than 0.08 or 0.06 for the RMSEA and SRMR support, respectively, acceptable and good model fit ( $\mathrm{Hu}$ and Bentler, 1999; Marsh et al., 2004). Convergent validity was assessed by inspecting the strength of items loading on their respective factors in the ESEM solution and discriminant validity was tested by showing that items presumed to load on one factor do not load on the others in the ESEM (Guay et al., 2015). In both bifactor CFA and bifactor ESEM, the maximum likelihood estimation with robust standard errors (MLR) is used.

\section{RESULTS}

The results shown in Table $\mathbf{1}$ indicate that the data have a relatively normal distribution. In addition, the correlation patterns seem to be within the expected direction. The CR values are reported in Table 2 based on the bifactor CFA and bifactor ESEM solutions.

The results of initial bifactor CFA and bifactor ESEM results in convergence issues of the solutions. The modification indices suggested that errors in two items on intrinsic orientation (item 1 and item 2) are highly correlated. The two items seem to be quite similar in wording, thus they appear to share similar measurement errors. After correlating the error terms, the two models converged. It appeared that the bifactor CFA yielded less than optimal fit to the data [i.e., $\chi^{2}(125)=334.516, p<0.05$, $\mathrm{CFI}=0.91$, TLI $=0.88$, RMSEA $[90 \% \mathrm{CI}]=0.09[0.08 ; 0.10]$,
SRMR $=0.07]$. In contrast, the bifactor ESEM appeared to fit the data well [i.e., $\chi^{2}(94)=204.798, p<0.05, \mathrm{CFI}=0.96$, TLI $=0.91$, RMSEA [90\% CI] $=0.07$ [0.06; 0.09], $\mathrm{SRMR}=0.03]$.

Although the bifactor ESEM outperforms its counterpart bifactor CFA, it was believed appropriate to provide the factor loadings of the two models for the sake of empirical comparison. As it can be seen in Table 2, the factor loadings in both solutions appear to reflect the idea of SDT theoretical underpinning. A key advantage of the bifactor ESEM, in addition to its better fit to the data, is that it accounts for the general factors adequately (i.e., autonomous motivation and controlled motivation) over the variance that already expressed through the specific factors (intrinsic, identified, introjected, and external regulations). That is, the loadings on the general factors were high and positive for the items related to its presumed general factors (for autonomous motivation the loadings ranged from 0.63 to 0.83 , and for controlled motivation, the loadings ranged from 0.01 to 0.70 ). Moreover, it appears that items on intrinsic and identified regulations loaded more strongly on the general factor "autonomous motivation" than on the specific factors. Except for one item (i.e., introjected 4) the items of "introjected regulation" loaded strongly on their specific factor. A similar observation is found among the items of "external regulation" in which only the loading of "external 4" was stronger on the general factor "controlled motivation" than on the specific factor.

Accordingly, we proceed with the bifactor ESEM to assess the predictive power of the SDT-L2 scale for the outcome. In the structural bifactor ESEM, the latent variable "L2 performance" was included as a dependent latent variable to examine the extent to which the two general factors (i.e., autonomous motivation and controlled motivation) predict the outcome as hypothesized. It should be noted that a bifactor ESEM where both the general and specific factors allowed to predict the outcome resulted in a nonconverged solution. Therefore, the author retain a bifactor ESEM model where the outcome was only predicted by the general factors. Table 3 shows that this model has yielded good model fit [i.e., $\chi^{2}(131)=265.722, p<0.05$, CFI $=0.94$, TLI $=0.90$, RMSEA $[90 \% \mathrm{CI}]=0.07[0.06 ; 0.09], \mathrm{SRMR}=0.05]$. The path coefficients of this model indicates that autonomous motivation strongly and positively predicted L2 performance $(\beta=0.87, p<0.001)$, while controlled motivation failed to predict L2 performance $(\beta=0.16, p>0.05)$. The variance explained in the outcome (i.e., L2 performance) was rather high $\left(R^{2}=0.89\right)$ indicating robust predictive power of the structural bifactor ESEM model. Although some would argue that the high value in $R^{2}$ is a result

TABLE 1 | Descriptive statistics and zero-order correlations for the variables.

\begin{tabular}{|c|c|c|c|c|c|c|c|c|c|}
\hline Variable & $M$ & $S D$ & Skew/Kurtosis & 1 & 2 & 3 & 4 & 5 & 6 \\
\hline 1. GPA & 4.07 & 1.12 & $-0.31 /-0.18$ & - & & & & & \\
\hline 2. Effort & 3.75 & 1.17 & $-0.25 /-0.16$ & $0.36^{\star \star \star}$ & - & & & & \\
\hline 3. Intrinsic regulation & 3.66 & 0.89 & $-1.56 / 2.34$ & $0.35^{\star \star \star}$ & $0.47^{\star \star \star}$ & - & & & \\
\hline 4. Identified regulation & 4.17 & 0.90 & $-1.87 / 3.19$ & $0.31^{\star \star \star}$ & $0.42^{\star \star \star}$ & $0.69^{\star \star \star}$ & - & & \\
\hline 5. Introjected regulation & 3.38 & 1.34 & $-0.42 /-1.12$ & $-0.18^{\star \star}$ & 0.01 & -0.03 & $0.17^{\star}$ & - & \\
\hline 6. External regulation & 4.23 & 0.86 & $-1.01 /-0.07$ & -0.11 & $-0.18^{\star \star}$ & 0.05 & 0.07 & $0.38^{\star \star \star}$ & - \\
\hline
\end{tabular}

${ }^{*} p<0.05,{ }^{* *} p<0.01,{ }^{* * *} p<0.001$ Examining the Factor Structure of the SDT-L2. 
TABLE 2 | Bifactor CFA and bifactor ESEM factor loadings of the SDT-L2 scale.

\begin{tabular}{|c|c|c|c|c|c|c|c|c|c|}
\hline \multirow[t]{2}{*}{ Items } & \multicolumn{3}{|c|}{ Bifactor CFA } & \multicolumn{6}{|c|}{ Bifactor ESEM } \\
\hline & Autonomous & Controlled & Specific factor & Autonomous & Controlled & Intrinsic & Identified & Introjected & External \\
\hline Intrinsic 1 & $0.82^{*}$ & & $0.54^{\star}$ & $0.80^{*}$ & & $0.26^{\star}$ & 0.02 & 0.05 & $-0.17^{\star}$ \\
\hline Intrinsic 2 & $0.59^{\star}$ & & $0.61^{\star}$ & $0.77^{*}$ & & $0.52^{\star}$ & 0.14 & 0.02 & 0.05 \\
\hline Intrinsic 3 & $0.75^{\star}$ & & $0.56^{\star}$ & $0.80^{\star}$ & & $0.43^{\star}$ & 0.07 & 0.07 & -0.16 \\
\hline Intrinsic 4 & $0.69^{*}$ & & $0.71^{*}$ & $0.72^{*}$ & & $0.21^{*}$ & 0.06 & -0.06 & 0.04 \\
\hline Intrinsic 5 & $0.79^{\star}$ & & $0.76^{\star}(0.85)$ & $0.83^{\star}$ & & 0.19 & 0.03 & 0.13 & -0.05 \\
\hline Identified 1 & $0.81^{\star}$ & & $0.63^{*}$ & $0.72^{\star}$ & & 0.11 & $0.34^{*}$ & -0.01 & 0.03 \\
\hline Identified 2 & $0.69^{*}$ & & $0.58^{*}$ & $0.63^{*}$ & & 0.18 & $0.25^{\star}$ & -0.07 & -0.06 \\
\hline Identified 3 & $0.74^{*}$ & & $0.56^{\star}$ & $0.71^{*}$ & & 0.06 & $0.60^{*}$ & 0.05 & -0.03 \\
\hline Identified 4 & $0.77^{\star}$ & & $0.52^{\star}$ & $0.68^{*}$ & & 0.01 & $0.65^{\star}$ & -0.03 & 0.06 \\
\hline Identified 5 & $0.69^{*}$ & & $0.53^{*}(0.89)$ & $0.64^{*}$ & & 0.09 & $0.56^{\star}$ & -0.02 & -0.08 \\
\hline Introjected 1 & & 0.14 & $0.75^{\star}$ & & 0.18 & 0.03 & $0.31^{*}$ & $0.66^{\star}$ & 0.09 \\
\hline Introjected 2 & & $0.25^{\star}$ & $0.75^{\star}$ & & $0.22^{\star}$ & 0.15 & 0.15 & $0.83^{*}$ & 0.04 \\
\hline Introjected 3 & & 0.13 & $0.66^{\star}$ & & 0.18 & -0.04 & -0.03 & $0.43^{*}$ & 0.16 \\
\hline Introjected 4 & & $0.28^{*}$ & $0.62^{*}$ & & $0.36^{\star}$ & 0.06 & -0.02 & 0.07 & -0.04 \\
\hline Introjected 5 & & 0.02 & $0.62^{\star}(0.67)$ & & 0.01 & -0.08 & 0.06 & $0.41^{*}$ & $0.28^{*}$ \\
\hline External 1 & & $0.32^{*}$ & $0.80^{\star}$ & & $0.22^{\star}$ & -0.09 & 0.09 & 0.09 & $0.80^{\star}$ \\
\hline External 2 & & $0.41^{\star}$ & $0.88^{*}$ & & $0.43^{\star}$ & -0.01 & -0.04 & 0.02 & $0.92^{*}$ \\
\hline External 3 & & 0.13 & $0.62^{\star}$ & & $0.35^{\star}$ & -0.07 & -0.07 & $0.32^{\star}$ & $0.37^{\star}$ \\
\hline External 4 & & $0.42^{*}$ & $0.61^{*}$ & & $0.70^{\star}$ & -0.04 & -0.01 & $0.43^{\star}$ & 0.16 \\
\hline External 5 & & $0.41^{*}$ & $0.63^{\star}(0.74)$ & & $0.43^{\star}$ & 0.08 & -0.04 & 0.12 & $0.62^{\star}$ \\
\hline $\mathrm{CR}$ & 0.84 & 0.82 & & 0.81 & 0.70 & 0.50 & 0.38 & 0.57 & 0.48 \\
\hline
\end{tabular}

${ }^{*} p<0.05$; CR values for the bifactor CFA specific factors are in parentheses.

TABLE 3 | Bifactor CFA, bifactor ESEM, and structural bifactor ESEM model fit indices for the SDT-L2.

\begin{tabular}{lccccc}
\hline Model & $x^{2}$ & df & SRMR & RMSEA (Low 90/Hi 90\%) & CFI \\
\hline Bifactor CFA & $334.516^{*}$ & 125 & 0.07 & $0.09(0.08 / 0.10)$ & 0.91 \\
Bifactor ESEM & $204.798^{*}$ & 94 & 0.03 & $0.07(0.06 / 0.09)$ & 0.88 \\
Structural bifactor ESEM & $265.722^{*}$ & 131 & 0.05 & $0.07(0.06 / 0.09)$ & 0.96 \\
\hline
\end{tabular}

${ }^{*} p<0.05$ : error terms of two items on intrinsic motivation have been correlated.

of overfitted model, this is, indeed, expected given the inherent complexity of the bifactor ESEM solution (e.g., the number of parameters estimated is larger than in standard CFA models).

\section{DISCUSSION}

The present study aimed at providing precise information about the factorial structure of the domain-specific scale, the SDTL2, by adopting the advanced framework of ESEM that allows for the combination of EFA, CFA, and SEM into a single assessment (Marsh et al., 2009; Morin et al., 2013, 2016; Alamer and Marsh, 2022). The researcher used the recently suggested model specification within the ESEM framework, that is the bifactor ESEM, to allow for the assessment of global factors (Howard et al., 2018). The current study demonstrated the usefulness of the bifactor ESEM for the language learning domain, and especially for L2 psychological scales by showing its application in testing the dimensionality of the SDT-L2 scale. In line with previous research, it appeared that bifactor
ESEM outperformed the bifactor CFA in the goodness-of-fit, owning to its flexibility in the analysis. Therefore, the theoretical framework of SDT has been supported in the bifactor ESEM model because of the inclusion of the two overarching constructs (i.e., autonomous motivation and controlled motivation), in which the items loaded properly on these general factors while also loaded on their specific factors (Ryan and Deci, 2017). This preposition was not possible to be accounted for in the EFA or the standard CFA models (Guay et al., 2015). Importantly, the findings of the present study contradict the high correlation between intrinsic regulation and external regulation reported in Ardasheva et al. (2012), and showed that the bifactor ESEM can be a more realistic representation of the measurement.

In addition, the present study has shown that bifactor ESEM can be used to provide evidence for convergent and discriminant validity from a new empirical perspective. That is, convergent validity was achieved by showing that items were strongly loaded on their hypothesized factors, even though they were allowed to cross-load on the other factors. Discriminant validity was 
achieved by showing that items presumed to load on one factor did not load substantially on the others in the ESEM solution, and if cross-loaded they presented weaker loading than on their presumed factor. Moreover, the present study was uniquely able to provide information on the predictive validity of the SDTL2 scale within the framework of ESEM. By turning the bifactor ESEM into a structural bifactor ESEM model we were able to gain precise and high-quality results of the role of the global constructs (i.e., autonomous motivation and controlled motivation) in predicting the outcome. Because of the flexibility of the bifactor ESEM model the explained variance was quite high (i.e., $89 \%$ of the variance in the outcome was explained by the variables in the bifactor ESEM model). In line with SDT literature, it was found that autonomous motivation positively and meaningfully related to an increase in L2 achievement, while controlled motivation failed to predict the outcome (Oga-Baldwin and Nakata, 2017; Alamer and Lee, 2019, 2021; Dincer et al., 2019; Noels et al., 2019).

Although the present study used an advanced method (i.e., bifactor ESEM) to evaluate the multidimensionality of SDT in the language learning domain, it has some limitations. First, the study used a relatively moderate sample size from one socio-cultural context, thus we would like to see replication of this study on other contexts using larger sample sizes. In addition, the present study relied on cross-sectional data. Future studies could benefit from using longitudinal data to account for the invariance of the measure over time.

Despite these limitations, the findings of the current research confirm the validity of the SDT-L2 scale to be used to assess students' L2 motivation from the SDT perspective. The study is the first to employ the bifactor ESEM in language learning domain and I hope that researchers apply it in

\section{REFERENCES}

Alamer, A. (2021). Basic psychological needs, motivational orientations, effort, and vocabulary knowledge: a comprehensive model. Stud. Second Lang. Acquis. 1-21. doi: 10.1017/S027226312100005X

Alamer, A., and Almulhim, F. (2021). The interrelation between language anxiety and L2 motivation; A mixed methods approach. Front. Educ. 8:618655. doi: 10.3389/feduc.2021.618655

Alamer, A., and Lee, J. (2019). A motivational process model explaining L2 saudi students' achievement of English. System 87:102133. doi: 10.1016/j.system.2019. 102133

Alamer, A., and Lee, J. (2021). Language achievement predicts anxiety and not the other way around: a cross-lagged panel analysis approach. Lang. Teach. Res. 1-22. doi: $10.1177 / 13621688211033694$

Alamer, A., and Marsh, H. (2022). Exploratory structural equation modeling in second language research: the case of the dualistic model of passion. Stud. Second Lang. Acquis.

Ardasheva, Y., Tong, S., and Tretter, T. (2012). Validating the english language learner motivation scale (ELLMS): pre-college to measure language learning motivational orientations among young ELLs. Learn. Individ. Differ. 22, 473483. doi: 10.1016/j.lindif.2012.03.001

Collier, J. (2020). Applied Structural Equation Modeling Using AMOS: Basic to Advanced Techniques. Abingdon-on-Thames: Routledge. doi: 10.4324/ 9781003018414

Dincer, A., Yeşilyurt, S., Noels, K., and Vargas Lascano, D. (2019). Selfdetermination and classroom engagement of EFL learners: a mixed-methods their psychometric research. Researchers are encouraged to use the ESEM (and bifactor ESEM) method for construct validation purposes. Going beyond the traditional CFA should help researchers assess their scales from a novel and more appropriate perspective.

\section{DATA AVAILABILITY STATEMENT}

The raw data supporting the conclusions of this article will be made available by the authors, without undue reservation.

\section{ETHICS STATEMENT}

The studies involving human participants were reviewed and approved by the Imam Mohammed Ibn Saudi Islamic University. The patients/participants provided their written informed consent to participate in this study.

\section{AUTHOR CONTRIBUTIONS}

The author confirms being the sole contributor of this work and has approved it for publication.

\section{SUPPLEMENTARY MATERIAL}

The Supplementary Material for this article can be found online at: https://www.frontiersin.org/articles/10.3389/fpsyg. 2021.732016/full\#supplementary-material

study of the self-system Model of motivational development. SAGE Open 9:2158244019853913. doi: 10.1177/2158244019853913

Gardner, R. (2010). Motivation and Second Language Acquisition: The SocioEducational Model. Frankfurt: Peter Lang.

Guay, F., Morin, S., Litalien, D., Valois, P., and Vallerand, J. (2015). Application of exploratory structural equation modeling to evaluate the academic motivation scale. J. Exp. Educ. 83, 51-82. doi: 10.1080/00220973.2013.876231

Howard, J., Gagné, M., Morin, A., and Forest, J. (2018). Using bifactor exploratory structural equation modeling to test for a continuum structure of motivation. J. Manag. 44, 2638-2664. doi: 10.1177/0149206316645653

Hu, L. T., and Bentler, P. M. (1999). Cutoff criteria for fit indexes in covariance structure analysis: conventional criteria versus new alternatives. Struct. Equ. Modeling 6, 1-55.

Marsh, H. W., Hau, K. T., and Wen, Z. (2004). In search of golden rules: comment on hypothesis-testing approaches to setting cutoff values for fit indexes and dangers in overgeneralizing $\mathrm{Hu}$ and Bentler's (1999) findings. Struct. Equ. Modeling 11, 320-341.

Marsh, H., Muthén, B., Asparouhov, T., Lüdtke, O., Robitzsch, A., Morin, A., et al. (2009). Exploratory structural equation modeling, integrating CFA and EFA: application to students' evaluations of university teaching. Struct. Equ. Modeling 16, 439-476. doi: 10.1080/10705510903008220

Morin, S., Arens, A., and Marsh, H. (2016). A bifactor exploratory structural equation modeling framework for the identification of distinct sources of construct-relevant psychometric multidimensionality. Struct. Equ. Modelling 23, 116-139. doi: 10.1080/10705511.2014.96 1800 
Morin, S., Marsh, H., and Nagengast, B. (2013). "Exploratory structural equation modeling," in Structural Equation Modeling: A Second Course, eds G. Hancock and R. Mueller (Charlotte, NC: Information Age), 395-436.

Muthén, L. K., and Muthén, B. O. (1998). Mplus User's Guide, 7thEdn. Los Angeles, CA: Muthén and Muthén.

Noels, K., Clément, R., and Pelletier, L. (1999). Perceptions of teachers' communicative style and students' intrinsic and extrinsic motivation. Mod. Lang. J. 83, 23-34.

Noels, K., Lascano, D., and Saumure, K. (2019). The development of selfdetermination across the language course: trajectories of motivational change and the dynamic interplay of psychological needs, orientations, and engagement. Stud. Second Lang. Acquis. 41, 821-851. doi: 10.1017/ S0272263118000189

Oga-Baldwin, Q., and Nakata, Y. (2017). Engagement, gender, and motivation: a predictive model for Japanese young language learners. System 65, 151-163. doi: 10.1016/j.system.2017.01.011

Reise, S., Moore, T., and Haviland, M. (2010). Bifactor models and rotations: exploring the extent to which multidimensional data yield univocal scale scores. J. Pers. Assess. 92, 544-559. doi: 10.1080/00223891.2010.49 6477

Ryan, R., and Deci, E. (2000). Self-determination theory and the facilitation of intrinsic motivation, social development, and well-being. Am. Psychol. 55, 68-78.

Ryan, R., and Deci, E. (2017). Self-Determination Theory: Basic Psychological Needs in Motivation, Development, and Wellness, 2nd Edn. New York, NY: Guilford Publications.
Ryan, R., and Deci, E. (2020). Intrinsic and extrinsic motivation from a selfdetermination theory perspective: definitions, theory, practices, and future directions. Contemp. Educ. Psychol. 61:101860. doi: 10.1016/j.cedpsych.2020. 101860

The Jamovi Project (2019). Jamovi (1.1) [Computer software]. Available online at: https://www.jamovi.org

Watanabe, M. (2011). Motivation, Self-Determination, and Willingness to Communicate by English Learners at a Japanese High School. Philadelphia, PA: Temple University.

Conflict of Interest: The author declares that the research was conducted in the absence of any commercial or financial relationships that could be construed as a potential conflict of interest.

Publisher's Note: All claims expressed in this article are solely those of the authors and do not necessarily represent those of their affiliated organizations, or those of the publisher, the editors and the reviewers. Any product that may be evaluated in this article, or claim that may be made by its manufacturer, is not guaranteed or endorsed by the publisher.

Copyright (c) 2021 Alamer. This is an open-access article distributed under the terms of the Creative Commons Attribution License (CC BY). The use, distribution or reproduction in other forums is permitted, provided the original author(s) and the copyright owner(s) are credited and that the original publication in this journal is cited, in accordance with accepted academic practice. No use, distribution or reproduction is permitted which does not comply with these terms. 